\title{
Directing Protein Assembly At Interfaces: Balancing Electrostatic And Hydrophobic Forces and the Role of Epitaxy.
}

\author{
Christopher M. Yip* \\ *Departments of Chemical Engineering and Applied Chemistry, and Biochemistry, Institute of \\ Biomaterials and Biomedical Engineering. University of Toronto 407 - 4 Taddle Creek Rd \\ Toronto, Ontario, Canada M5S 3G9
}

The rational design of protein-based supramolecular architectures requires careful consideration of not only intramolecular structure but also the intermolecular interactions that control their self-association into higher order structures. We are particularly interested in the role of interfacial structure and chemistry in defining the nucleation and growth of these systems and specifically the synthesis of extended two-dimensional protein arrays. Extended-duration in situ tapping mode atomic force microscopy was employed to investigate the role of electrostatic and hydrophobic forces on the assembly of $\beta$-helix and $\beta$-sheet forming peptides at ordered interfaces.

While a 33-residue fragment of the hexapeptide LPX-A rapidly self-associated on mica to form well-defined rectangular rod structures in epitaxy with the (001) surface of mica, on highly oriented pyrolytic graphite, we only observed an amorphous $8 \AA$ thick film. This is in stark contrast to the results obtained for a model hexapeptide repeat (NAKIGD) that was found to assembly epitaxially on both mica and HOPG. Similar studies performed with insulin revealed epitaxial growth of two-dimensional fibril domains on HOPG but only amorphous aggregation on mica.

Protein assembly is critically dependent not only on the amino acid sequence but also the conformation and relative exposure of specific functional domains. This is of particular importance for understanding the mechanisms by which proteins form coherent supramolecular architectures, such as extended beta-sheet or beta-helical fibres. Such a motif has been proposed to underlie the unique mechanical and physical properties of the extracellular protein, elastin, which can undergo reversible gelation, or coacervation, at elevated temperatures [1]. We report here the results of a variable temperature in situ atomic force microscopy study of the assembly of recombinant human tropoelastin proteins EP I and EP II at ordered interfaces. On mica, both of these peptides assemble as discrete stable globular aggregates. However, on highly ordered pyrolytic graphite (HOPG), the assembly process proceeds via the initial formation of an twodimensional film and subsequent nucleation of high-aspect ratio rods on this prelayer [Figure 1] Remarkably these aggregates appear to form in an ordered epitaxial relationship with the underlying hexagonal lattice of the (0001) cleavage plane of HOPG. For EP I and II, the surface transition temperature was higher than the known solution coacervation temperatures suggesting that surface confinement of the peptide presents an additional energetic barrier to fibril nucleation that is not present in solution [Figure 2].

These unique characteristics provide an intriguing design strategy for the creation of novel peptide-based nanostructures. Our results suggest that careful manipulation of surface electrostatics and hydrophobics can be used to control protein assembly at interfaces [2] 
[1] C.M. Bellingham et al., Biochim Biophys Acta 1(2001), 6

[2] This work was supported by the Canadian Institutes of Health Research (MT-14769),

NSERC (194435-99), the Canada Foundation for Innovation, the Ontario Research and

Development Challenge Fund, and the Ontario Innovation Trust. C. M. Y. is a Canada Research

Chairs Program Chairholder.

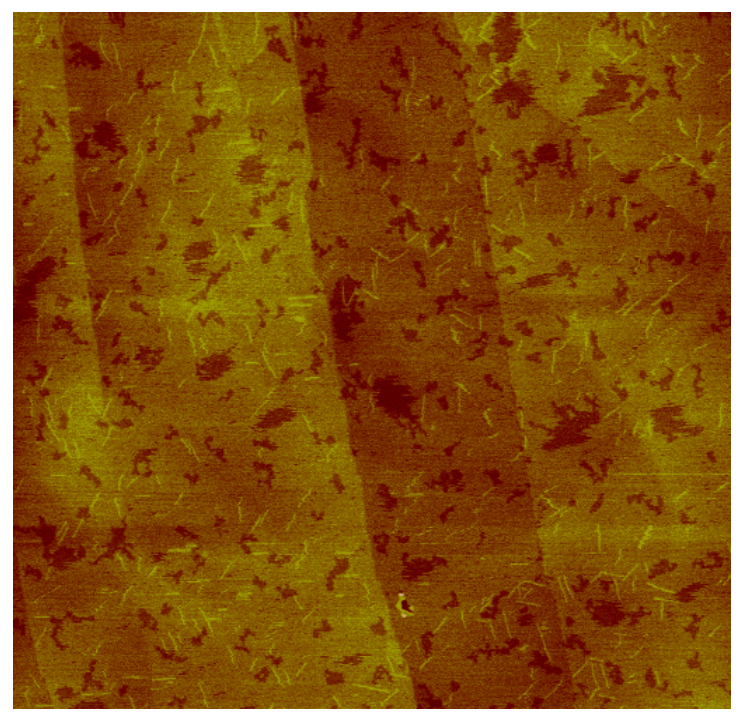

Figure 1a. In situ tapping mode AFM image of EPI peptide on HOPG acquired in Tris buffer solution at $50^{\circ} \mathrm{C} 30$ minutes after peptide addition. Note that fibril formation has begun and is in alignment with underlying substrate. Image size: $4 \mu \mathrm{m} \times 4 \mu \mathrm{m}$

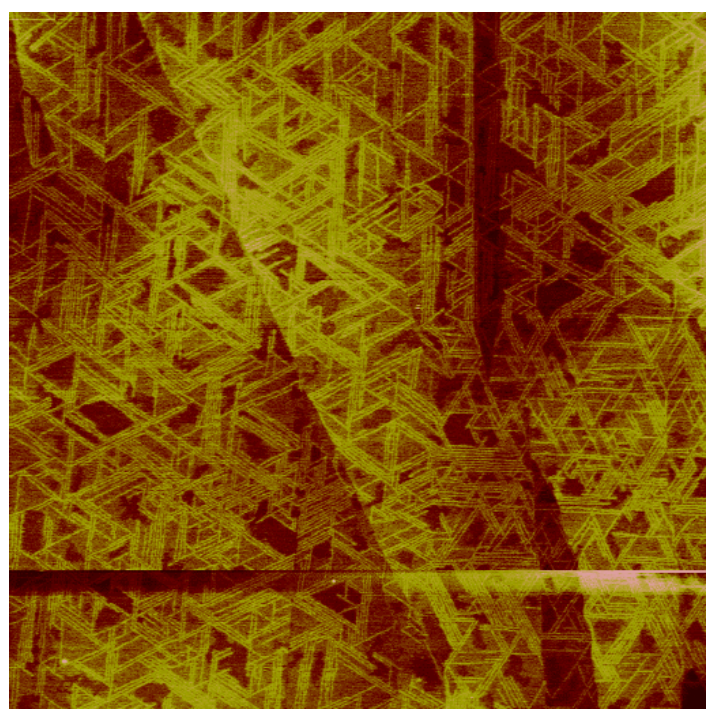

Figure 1b. In situ tapping mode AFM image of EPI peptide on HOPG acquired in Tris buffer solution at $50^{\circ} \mathrm{C}$ approximately $2 \mathrm{hrs}$ after the initial addition of the peptide. Note the high density of $\sim 1 \mathrm{~nm}$ thick fibres on the surface. Image size: $4 \mu \mathrm{m}$ x $4 \mu \mathrm{m}$

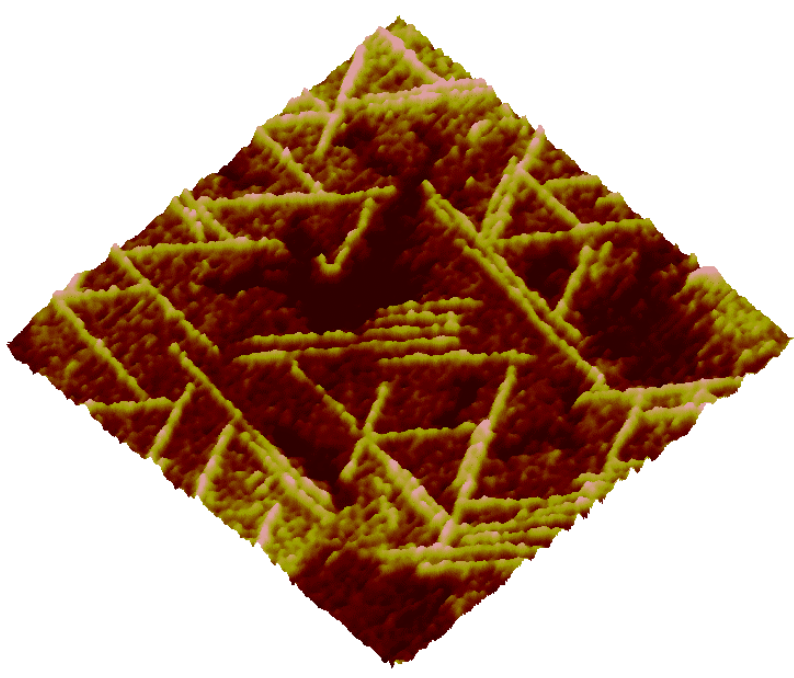

Figure 2a. In situ tapping mode AFM image of EPI peptide on HOPG acquired in Tris buffer solution at $50^{\circ} \mathrm{C}$. This image clearly reveals the complex nature of the EP I multilayer assembly Image size: $1 \mu \mathrm{m}$ x $1 \mu \mathrm{m}$ 